Biol Neonate 1995;68:431-432

\title{
Subject Index Vol. 68, 1995
}

Abdominal masses 175

Absorption 354

Acetyl-CoA carboxylase 128

Acute life-threatening episodes 87

Adenosine Ill

Alveolization 229

Angiography 75

Animals 185

Antioxidant 1

Argininosuccinate lyase 221

Ascorbic acid 1

ATP citrate lyase 128

Atrial extract 292

Autonomic nervous system 270

Bilirubin 135,301

kinetics 135 Birth weight 10 Blood pressure 318 Body composition 384 Bone mineral contents 254

- status 254 Brain 135

blood flow 200

cilia 394

Caffeine 169

Calcitriol 157

Calciuria 169

Calf 354

Capillary maturation 229

Carnitine 211

- depletion 211

Catecholamine 404

CD26 antigen 259

CD31 antigen 259

Cerebral blood flow/oxygenation 91

circulation, pig 75

cortex 419

vascular bed 200 Chromatography 292 Claustrum 47

Clinical pharmacology 276 Colostrum 15,259,354

Cord blood 1

- $\quad$ - monocytes 308

Cordocentesis 163 
Cyclooxygenase byproducts 342

Cystic masses 175

Delivery mode 404 Dendritic development 47 Development Ill,141 Developmental toxicity 62

Dexamethasone 39 Diabetes 377 Disaturated phosphatidylcholine

39 DNA synthesis 157 Dobutamine 318 Dopamine 318 Doppler ultrasonography 318

- $\quad$ ultrasound 163

Dual-energy X-ray absorptiom-

etry 254 Dwarfism 412

Encephalopathy 135 ß-Endorphins 87 Enzyme-linked immunosorbent

assay 185 Ependymal cilia 394 Epidermal growth factor 26 Eutectic mixture, lidocaine/prilo-

caine 334

Fatty acid synthase 128 Fetal blood 163

hepatocytes, primary culture 221

jejunum 157

lamb, cerebral circulation 200

- $\quad$ rat lung 39

Flavobacterium meningosepticum

153 Flow microfluorometry 308 Focal ischemia 75 Functional residual capacity 246 Furosemide, inhaled 191

Gastroesophageal reflux 87 Glucocorticoid(s) 221,229 Glucose metabolism 26

- $\quad$ tolerance 282

Granulocyte-macrophage colony-

stimulating factor 104

Growth hormone deficiency 412 Guinea pig 191

Host defense 308 Human 157

fetus 377

milk 325

- macrophages 325 Hypotension 318 Hypoxia-induced vasodilatation

200

Immune response 153 Indomethacin 342

- $\quad$ infusion 91

Infant total-body electrical

conductivity 384 Insulin 384 Insulin-like growth factor(s) 62

- factor-1 354,368

tolerance 282 Interferon- $\gamma 104$ Interleukin-1ß 104 Interleukin-6 104 Intestinal perfusion 318

Intestine 354 Intracellular bilirubin 100 Intratracheal instillation 191 Intrauterine growth

retardation

$19,163,377,412$

Kernicterus 135 Kidney 141

Labour 104

Lactase 157

Left ventricular output 404

Lipid extract surfactant 301

Lipoprotein 119

431

Local anesthesia, newborn 
animals 334 Lumbar spine 254 Lung development 229

- $\quad$ interstitium 229

Lymphocytes 100

Malformation, rats 33 Malic enzyme 128 Maternal toxicity 62 Membrane(s) 141

antigens 308

receptors 308 Meningitis 153 Messenger RNA 221 Metatarsal bone 368 f15N]Methacetin 19

Methamphetamine 33 Methemoglobin concentration

334 Monocytes 100 Monooxygenase activity 19 Morphometry 229 Mother 104 Multicystic

dysplasia 175

$\mathrm{Na}+, \mathrm{K}+$-ATPase 419 Necrotizing enterocolitis 81,318 Neonatal diseases 334

growth 26

jaundice 100,398

respiratory cilia 394 Neonates 153 Neonatology 354 Nephroblastoma 175 Neutrophils 100,264

Newborn(s) 104

piglets 419

-, preterm, term 1 -, pups 119 Nitric oxide 200

- $\quad$ - synthase inhibition 419

NG-Nitro-L-arginine 419

Obesity, pregnancy 384 Organ blood distribution 404

Osteocalcin 254, 377 Osteopenia 254

Paediatrics 276 Pancreas 26 Pancreatic insulin 282 Parasympathetic activity 270 Parathyroid hormone 368 Penile circumcision, anesthetics

$334 \mathrm{pH} 301$

Phagocytosis 15,264 Pharmacokinetics 191 -, lidocaine/prilocaine 334 Phototherapy 398 Piglets 342 Pivalate 211 Plasma 292

Poly cystic kidney disease 175 Porcine growth hormone, fetal/

neonatal development 62 Postnatal development 119 Prealbumin 10 Pregnancy 282 Premature infant 246

neonate 264 Preterm babies 91

infant(s) 169,318

newborns 81 Preterm-born children 254 Progesterone 128 Protein hydrolysates 55 Pulmonary

function 342

surfactants 185

rANP 292

Rapid eye movement sleep

270 Rat(s) 55,282,412 -, adult 292 Receptor(s) Ill

expression 264 Regional clearance 135 Renal function 169

tumor 175

vein thrombosis 175

Respiratory distress syndrome 39, 81,185,246

- mechanics 246

Resting tone 200

Sepsis 342

Serum amino acids 55

Somatostatin 81

Sucrase 157 
Sudden infant death syndrome 87

Superior mesenteric artery 318

Superoxide anion 104

Surface tension 301

Surfactant 246

$\operatorname{protein}(\mathrm{s}) 301$

- A 185

- -, BandCmRNA 39 Sympathetic activity 270

T lymphocytes 259 Theophylline 169 Thyrotropin-releasing hormone

39 Transcutaneous bilirubinometry

398 Transforming growth factor- $\alpha 325$ Transthyretin 10 Tumor necrosis factor 342 Tumour necrosis factor- $\alpha 104$

Ukraine 276 Umbilical artery 163 Undergraduate medical education

276 Undernutrition, rats 47

Vasoconstriction 318 Venous flow 163 Vessel anatomy 75

Whole-embryo culture 33

432

Subject Index Vol. 68, 1995 